\title{
In-situ Observation of Shear Deformation of Gold Single Real Contact Point at the Nanoscale
}

\author{
Tadashi Ishida*, Takaaki Sato and Hiroyuki Fujita \\ Center for International Research on Micronano Mechatronics, Institute of Industrial Science, University of Tokyo \\ 4-6-1 Komaba, Meguro-ku, Tokyo 153-8505, Japan \\ *Corresponding author: tadashii@iis.u-tokyo.ac.jp
}

( Manuscript received 1 January 2012; accepted 5 June 2012; published 30 September 2012 )

(Presented at Symposium S3: Science of Friction: Towards Realization of Society with Carbon Minimum in the International Tribology Conference Hiroshima 2011)

\begin{abstract}
We in-situ observed the deformation of a gold single real contact point at the nanoscale during friction experiment in order to reveal the mechanism of friction. In this experiment, a special experimental setup combining between micro-electro-mechanical system (MEMS) and transmission electron microscope (TEM), called MEMS-in-TEM, was utilized for the stable visualization of the real contact point. Gold tips with electrostatic actuators were gently brought into contact and were rubbed each other at a quasi-static speed under TEM observation. Between tips, the gold single real contact point was formed and deformed in shear. During this deformation, the ends of the real contact point travelled $2.8 \mathrm{~nm}$ along the surface of the gold tips, although those of silicon and silver did not move. The maximum friction was $60 \mathrm{nN}$, repeating the stick-slip behavior from 35 to $60 \mathrm{nN}$. This result will be helpful to understand the mechanism of friction at the nanoscale and the lubrication mechanism of solid lubricants.
\end{abstract}

Keywords: gold, in-situ observation, real contact point, micro-electro-mechanical systems, transmission electron microscope

\section{Introduction}

Joints and interfaces in mechanical components, such as engines and gears, always cause friction and wear. Such tribological issues are quite important to estabilish carbon minimum society, because friction causes extremely large amount of energy loss in mechanical devices and wear shorten the lifetime of mechanical devices. Many studies have been done for the reduction of friction and wear, with changing the material combination and surface condition [1-3]. Up to now, the mechanism of friction and wear is explained by Amonton-Coulomb's law; friction is caused by the real contact points formed between the surface asperities [4]. However, many friction phenomena cannot be explained just by the law. Therefore, the mechanism of the friction is not well understood. In order to fully understand friction, which is the macro-scaled physics, it is important to study friction from the nanoscale and even the atomic scale, leading to the elucidation of the friction.

Scanning probe microscopes (SPM), such as atomic force microscope (AFM) [5] and friction force microscope (FFM) [6], are good approaches to study friction at the nanoscale. This is because the radius of curvature of the probe apex is a few nm to sub nm, resulting in the friction information from the nanometer and even atomic scale area. Stick-slip motion at the atomic scale was observed [7] and ultra small friction less than $\mathrm{nN}$ was measured [8] using SPM. However, the behavior at the interface between the probe and the sample surface could not be observed during the friction experiment. It is important that the difference of the deformation behavior of the real contact point largely affects the frictional properties. AFM was installed into a transmission electron microscope (TEM) to observe the interface behavior at the nanoscale [9]. Using this experimental setup, it was found that the nanoscaled contact point deformed during the friction experiment and the deformation of the contact point caused friction [10]. This result is well matched to the recent explanation of Amonton-Coulomb's law. In such experimental setup, the AFM probe was actuated by goniometers and piezo actuators. The drawback of these actuators is unstability; charging and thermal drifts and the mechanical viblation, which prevent the observation of the nanoscaled contact. In order to solve this problem, we establish the experimental setup of the combination between micro-electro-mechanical systems (MEMS) 
TEM

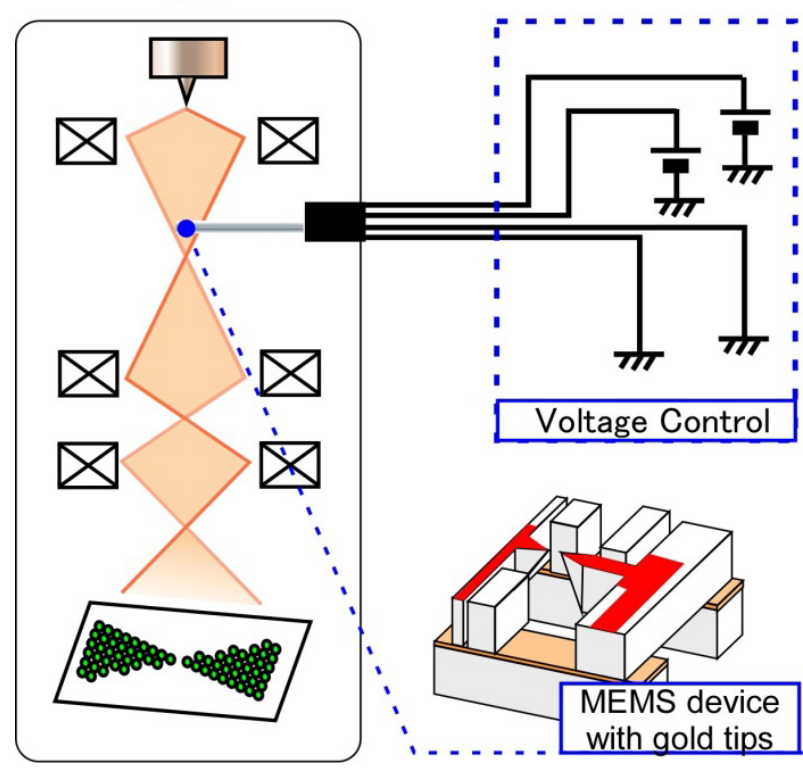

Fig. 1 Experimental setup, MEMS-in-TEM. MEMS device with gold opposing tips can be installed and driven inside a TEM specimen chamber with a TEM special sample folder. The ultra high vacuum condition of the TEM specimen chamber keeps the MEMS device clean.

and TEM, called MEMS-in-TEM [11]. Using this setup, the deformation of the real contact point of silicon and silver was in-situ observed during the friction experiment [12, 13]. In this report, we observed the deformation of a gold real contact point at the nanoscale during the friction experiment.

\section{Experimental}

We performed a friction experiment using MEMS-in-TEM for the visualization of nanoscaled deformation of a real contact point (Fig. 1). MEMS and TEM are the key technologies in this experimental setup.

\subsection{Experimental setup}

Figure 2 shows the MEMS device with gold opposing tips. The MEMS device has electrostatic actuators of 2 degrees of freedom; one is for an approach direction and another is for a friction direction [13]. These actuators are driven by electrostatic force in approach and friction directions when voltages are applied between a movable electrode and a driving electrode. The electrostatic actuators achieve quite precise displacement control within sub-nm order. Additionally speaking, the electrostatic actuator is superior to the piezoelectric actuator from the point of stability issue; it has quite small temporal, thermal and charging drift less than a few nm over several tens of minutes [14]. The spring constants of the movable electrodes are $237 \mathrm{~N} / \mathrm{m}$ for approach and $140 \mathrm{~N} / \mathrm{m}$ for friction, respectively.

TEM (Hitachi, HF-2000UHV) is used for the

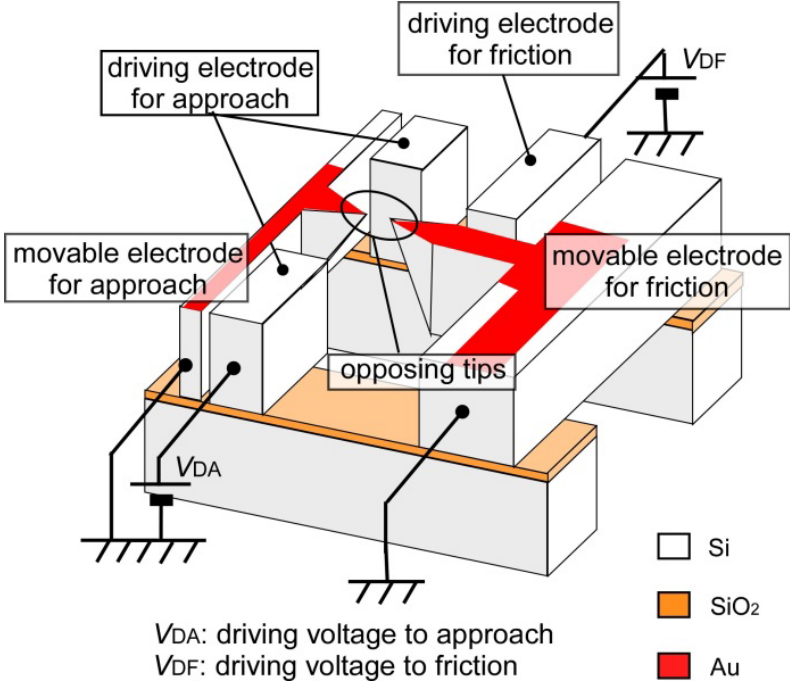

Fig. 2 MEMS device with gold opposing tips. One tip moves in approach direction and another moves in friction motion using electrostatic actuator, applying driving voltages to 2 degrees of freedom.

nanoscopic realtime observation of the real contact point at the nanoscale. Electron beam is extracted from a cold field emission type electron gun and is accelerated up to $200 \mathrm{kV}$ with $1.6 \times 10^{4} \mathrm{~A} / \mathrm{m}^{2}$. The spatial resolution was $0.2 \mathrm{~nm}$ and the time resolution was $1 / 30 \mathrm{~s}$. It has an ultra-high vacuum specimen chamber, $5 \times 10^{-8} \mathrm{~Pa}$, to keep the sample surface clean. The MEMS device is inserted into the specimen chamber using a specially customized sample holder for MEMS device. This TEM sample holder has 9 feedthroughs to apply voltage to the electrostatic actuators. The motions of opposing tips are controlled, with changing the voltage. The friction experiment inside TEM can be achieved with this special experimental setup. The temperature is room temperature because the temperature rise due to the electron beam irradiation can be neglected according to the thermal calculation [15].

\subsection{Force measurement between tips}

During this experiment, force applied to the contact point between tips can be calculated. The force is measured by the similar method as a force curve measurement in AFM, just by the product between the spring constant of AFM cantilever and displacement [16]. The displacement of the tip is measured by the TEM observation. First, we find some markers on tips which did not deform during the experiment. Then, the displacement of the tip without contact is measured by the observation, which is defined just by the driving voltage. On the other hand, the displacement of the tip with contact is measured. It should be smaller than that without contact at the same driving voltage, because the contact pulls the tip against its motion. The difference of the displacement with and without contact is obtained. 
(a) approach

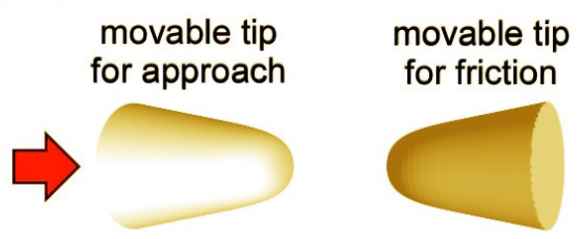

(b) formation

(c) friction

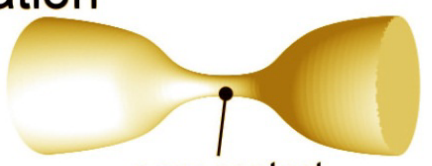

nanocontact

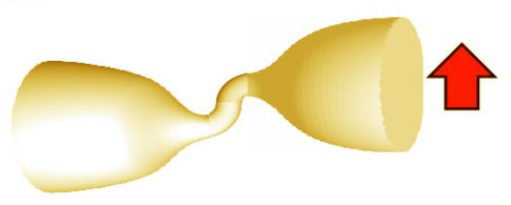

(d) fracture

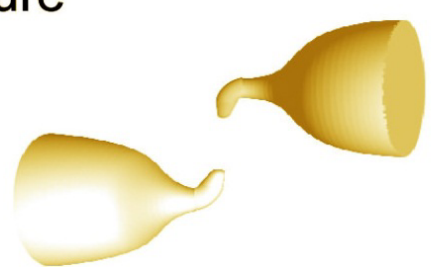

Fig. 3 Procedure of friction experiment using MEMS-in-TEM. (a) approach, (b) formation of gold single real contact point, (c) frictional deformation of the gold single real contact point, (d) fracture of the real contact point.

The applied force is calculated as the product between the difference of the displacement and the spring constant of the movable electrode [14]. Considering the spatial resolution of TEM, the force measurement resolution was $47.4 \mathrm{nN}$ for approach and $28 \mathrm{nN}$ for friction.

\subsection{Friction experiment using MEMS-in-TEM}

Figure 3 shows the experimental procedure of the friction experiment using MEMS-in-TEM. Gold tips face each other; one is for approach and another is for friction. The approach tip moves towards another tip. They are brought into gentle contact. A gold real contact point at the nanoscale is formed by this physical contact. The friction tip moves at the speed of $0.1 \mathrm{~nm} / \mathrm{s}$ perpendicular to the approach direction, resulting in the shear deformation of the gold real contact point. The gold nano real contact point is finally fractured.

\section{Results and Discussions}

Shear deformation and travel motion of the gold real contact point were observed during the friction experiment. Figure 4 shows the deformation of the gold
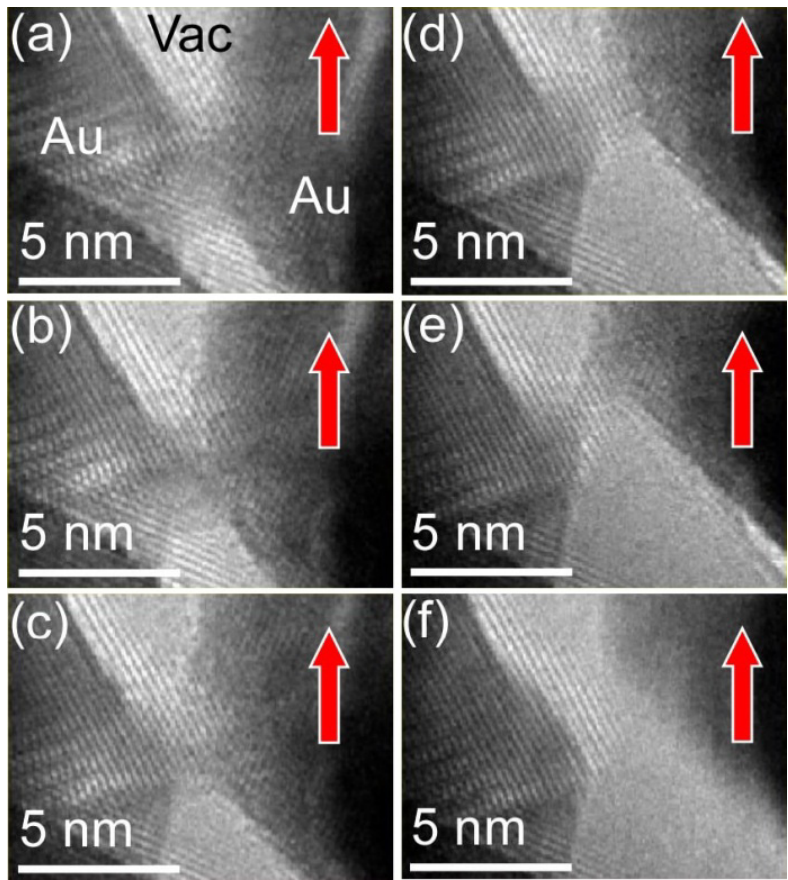

Fig. 4 In-situ TEM observation of shear deformation at gold real contact point. The gold real contact point gradually became thinner and thinner; (a) $3.8 \mathrm{~nm}$ at the initial position, (b) $3.3 \mathrm{~nm}$ at 4.0 $\mathrm{nm}$ in displacement, (c) $3.1 \mathrm{~nm}$ at $6.0 \mathrm{~nm}$, (d) 2.7 $\mathrm{nm}$ at $8.0 \mathrm{~nm}$, (e) $1.2 \mathrm{~nm}$ at $8.8 \mathrm{~nm}$. (f) It was finally fractured at $9.0 \mathrm{~nm}$ in displacement.

real contact point during the friction experiment taken by TEM. Gold tips with electrostatic MEMS actuators were gently brought into contact and a gold single real contact point of $3.8 \mathrm{~nm}$ in diameter was formed between tips (Fig. 4a). The normal force applied to the gold single real contact point was less than $47.4 \mathrm{nN}$, because the force resolution was $47.4 \mathrm{nN}$ and we could not detect the displacement difference with and without contact. The space between fringe images was $0.28 \mathrm{~nm}$, which corresponds to $\left(\begin{array}{lll}1 & 1 & 0\end{array}\right)$. One tip moved towards friction direction at the speed of $0.1 \mathrm{~nm} / \mathrm{s}$. The gold real contact point was deformed in shear motion; the diameter of the neck gradually decreased $3.3 \mathrm{~nm}$ at 4.0 $\mathrm{nm}$ in displacement (Fig. 4b), $3.1 \mathrm{~nm}$ at $6.0 \mathrm{~nm}$ (Fig. 4c), $2.7 \mathrm{~nm}$ at $8.0 \mathrm{~nm}$ (Fig. 4d), respectively. The gold real contact point was thinner and thinner after $8.3 \mathrm{~nm}$ in displacement; the diameter became $1.2 \mathrm{~nm}$ at $8.8 \mathrm{~nm}$ in displacement (Fig. 4e). It was finally fractured at $9.0 \mathrm{~nm}$ in displacement (Fig. 4f).

In addition to this shear deformation, the root of the real contact point moved along the surface of the gold tip. To detect the shear displacement, the middle points of the root of the single real contact were tracked (Fig. 5 ). The edges of gold opposing tips before contact were extrapolated and the middle points of the extrapolated edges were defined as roots of the gold single real contact point. We measured the travelling distance along 
(a) before

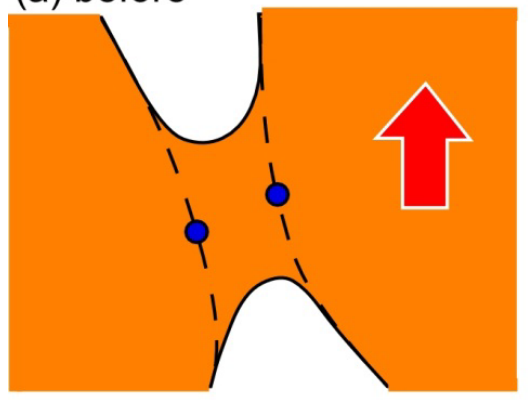

-- root of single real contact point (b) after

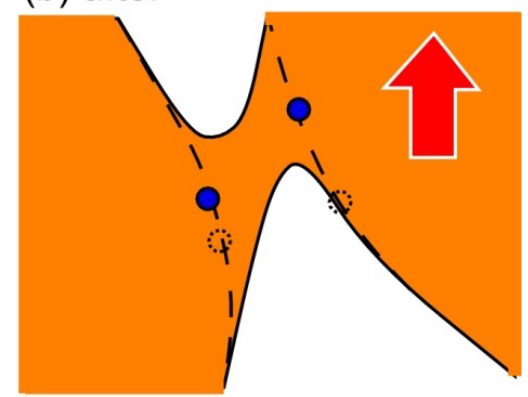

middle point of root line

Fig. 5 Schematic illustration of gold real contact point at the nanoscale before and after the friction experiment. (a) The gold real contact point bridging between opposing tips before the friction experiment. The edges of gold opposing tips before contact were extrapolated and the middle points of the extrapolated edges were defined as roots of the gold single real contact point. (b) The gold real contact point after the friction experiment. The middle points at the root were tracked during the experiment to measure the travelling distance of the root.
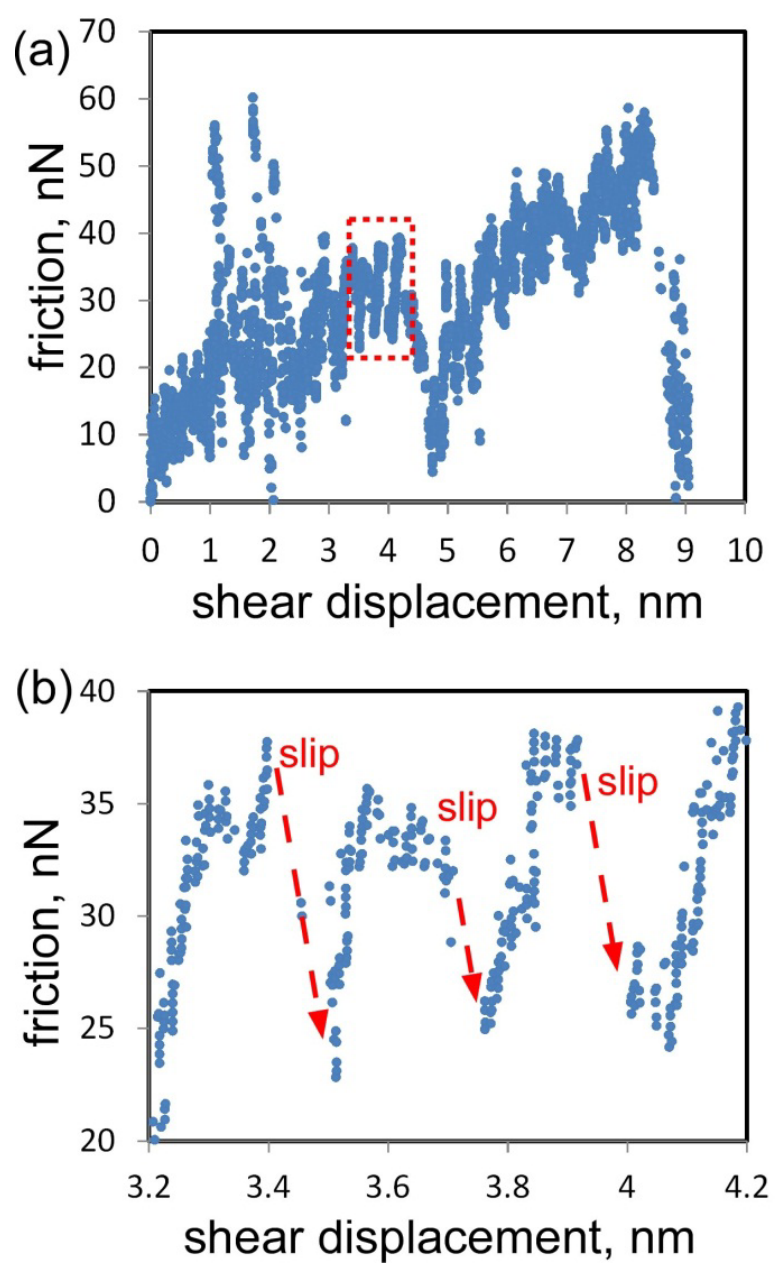

Fig. 6 Graph of friction as a function of shear distance. (a) The force gradually increased up to $60 \mathrm{nN}$. The force was kept from 35 to $60 \mathrm{nN}$ during the shear motion. The force decreased to zero after $8.3 \mathrm{~nm}$ in displacement. (b) the stick-slip motion during the shear motion. the surface by tracking the middle points of the root. The travel distance was $2.8 \mathrm{~nm}$. This motion of the root have never observed in silicon and silver $[12,13]$.

Figure 6a shows the friction curve as a function of shear displacement. The force increased from 0 to 55 $\mathrm{nN}$ while one tip moved to friction direction at 0.01 $\mathrm{nm} / \mathrm{s}$. After that, the force suddenly dropped to $7 \mathrm{nN}$ and then gradually increased up to $60 \mathrm{nN}$. In the increasing step, atomic scale drops of the friction were measured and seem stick-slip motion at the atomic scale (Fig. 6b, zoom-up graph of Fig. 6a from $3.2 \mathrm{~nm}$ to $4.2 \mathrm{~nm}$ ). This drop-increase cycle of the force was repeated with the peak values from 35 to $60 \mathrm{nN}$, before the shear displacement reached $8.3 \mathrm{~nm}$. After $8.3 \mathrm{~nm}$, the diameter of the real contact point decreased and the force also decreased. At $9.0 \mathrm{~nm}$, the gold real contact point was fractured. The maximum force was $60 \mathrm{nN}$ during this experiment.

The motion of the root of the gold single real contact point released the shear force applied to the contact during friction test. Due to this motion, the shear force cause not only the deformation of the gold single real contact point itself but also the motion of the root. As a result, gold could stand at higher shear force in comparison to the silver case. For the stick-slip motion during the friction test of the gold single real contact point, the displacement to induce the stick-slip motion was from 0.23 to $0.25 \mathrm{~nm}$. This value was almost similar to the lattice distance in ( $\left.\begin{array}{lll}1 & 1 & 1\end{array}\right)$, which is the slip plane in gold crystal. This result suggests that the stick-slip motion was caused by the crystalline structure.

\section{Conclusion}

The shear deformation of the gold real contact point was in-situ visualized during the friction experiment between gold MEMS opposing tips. We found that the motion at the root of the gold real contact point along the surface. This motion should have some relationship 
to the friction property and may be the key issue of the gold friction to elucidate the mechanism of friction.

\section{Acknowledgement}

This work was supported by KAKENHI; the grant-in-aid of Specially Promoted Research 21000008 sponsored by Japan Society for the Promotion of Science (JSPS).

\section{References}

[1] Spalvins, T., "A Review of Recent Advances in Solid Film Lubrication," Journal of Vacuum Science and Technology, A, 5, 1987, 212-219.

[2] Bhushan, B. and Jung, Y. C., "Micro- and Nanoscale Characterization of Hydrophobic and Hydrophilic Leaf Surfaces," Nanotechnology, 17, 2006, 2758.

[3] Kalin, M., Velkavrh, I. and Ozbolt, L., "Review of Boundary Lubrication Mechanisms of DLC Coatings Used in Mechanical Applications," Meccanica, 43, 2008, 623-637.

[4] Bhushan, B., "Introduction to Tribology," John Wiley and Sons Inc., New Jersey, 2002.

[5] Binnig, G., Quate, C. F. and Gerber, C., "Atomic Force Microscope," Physical Review Letters, 56, 1986, 930-933.

[6] Mate, C. M., McClelland, G. M., Erlandsson, R. and Chiang, S., "Atomic-Scale Friction of a Tungsten Tip on a Graphite Surface," Physical Review Letters, 59, 1987, 1942-1945.

[7] Howald, L., Haefke, H., Lüthi, R., Meyer, E., Gerth, G., Rudin, H. and Güntherodt, H.-J., "Ultrahigh-Vacuum Scanning Force Microscopy: Atomic-Scale Resolution at Monatomic Cleavage Steps," Physical Review B, 49, 1994, 5651-5656.

[8] Fujisawa, S., Kishi, E., Sugawara, Y. and Morita, S., "Two-Dimensionally Quantized Friction Observed with Two-Dimensional Frictional Force Microscope," Tribology Letters, 1, 1995, 121-127.
[9] Kizuka, T., "Atomic Process of Point Contact in Gold Studied by Time-Resolved High Resolution Transmission Electron Microscopy," Physical Review Letters, 81, 1994, 4448-4451.

[10] Fujisawa, S. and Kizuka, T., "Lateral Displacement of an AFM Tip Observed by In-Situ TEM/AFM Combined Microscopy: The Effect of the Friction in AFM," Tribology Letters, 15, 2003, 163-168.

[11] Ishida, T., Nakajima, Y., Kakushima, K., Mita, M., Toshiyoshi, H. and Fujita H., "Design and Fabrication of MEMS-Controlled Probes for Studying the Nano-Interface under in situ TEM Observation," Journal of Micromechanics and Microengineering, 20, 2010, 075011.

[12] Ishida, T., Sato, T., Nabeya, S. and Fujita, H., "Quasi-Static Frictional Test between Silicon Sharp Probes with in-situ TEM Observation of Real Contact Point," Journal of Physics: Conference Series, 258, 2010, 012011.

[13] Sato, T., Ishida, T., Nabeya, S. and Fujita, H., "Nano-Scale Observation of Frictional Deformation at $\mathrm{Ag}$ single Point Contact with MEMS-in-TEM Setup," Journal of Physics: Conference Series, 258, 2010, 012005.

[14] Sato, T., Ishida, T., Jalabert, L. and Fujita, H., "Development of MEMS-in-TEM Setup to Observe Shear Deformation for the Study of Nano-Scale Friction," Tribology Online, 6, 2011, 226-229.

[15] Ishida, T., Sato, T., Nabeya, S., Kakushima, K. and Fujita, H., "Highly Stable Spatio-Temporal Mechanical Characterization of Nanocontact between Sharp Tips Using Electrostatic Microactuator inside Transmission electron Microscope,” Japanese Journal of Applied Physics, 50, 2011, 077201.

[16] Iijima, S., "Microclusters," ed. Sutano, S, Nishina, Y. and Ohnishi, S., Springer-Verlag, Berlin, 1982, 186-192. 